\title{
ON THE EXPANSION OF A FUNCTION ANALYTIC AT DISTINCT POINTS
}

BY P. W. KETCHUM

1. Introduction. Let $a_{1}, a_{2}, \cdots, a_{\nu}$ be a set of distinct points in the complex plane.* If $\theta_{k}(x)$ is any function which is analytic at $a_{k}$ and has a simple zero at $a_{k}$, then for any sufficiently small positive number $\delta$ the level curve $\left|\theta_{k}(x)\right|=\delta$ will have one branch, $C_{\delta}(k)$, which satisfies the conditions (a) $a_{k}$ is interior to the curve $C_{\delta}{ }^{(k)}$, (b) $C_{\delta}{ }^{(k)}$ is a simple closed analytic curve, (c) $a_{k}$ is the only zero of $\theta_{k}(x)$ either inside or on $C_{\delta}^{(k)}$. Furthermore, if $\delta^{\prime}$ and $\delta^{\prime \prime}$ are any two values of $\delta$ such that $\delta^{\prime \prime}>\delta^{\prime}$, then $C_{\delta^{\prime}}(k)$ contains $C_{\delta^{\prime}}(k)$ in its interior.

Let $F_{n, m}(x),(n=0,1,2, \cdots ; m=1,2, \cdots, \nu)$, be a set of functions all of which are holomorphic in and on each of the curves $C_{R}{ }^{(k)}$, and let each function have a zero of order $n$ at $a_{m}$ and a zero of higher order than $n$ at all the other points $a_{k}$, $(k \neq m)$. Then in and on $C_{R}{ }^{(m)}$, we have the Bürmann series $\dagger$

$$
F_{n, m}(x)=\sum_{s=0}^{\infty} c_{n, s}^{(m, m)}\left[\theta_{m}(x)\right]^{n+s} \equiv\left[\theta_{m}(x)\right]^{n} P_{n, m, m}(x),
$$

where $c_{n, 0}^{(m, m)} \neq 0$. In and on $C_{R}^{(k)},(k \neq m)$, we have

$$
F_{n, m}(x)=\sum_{s=0}^{\infty} c_{n, s}^{(m, k)}\left[\theta_{k}(x)\right]^{n+s+1} \equiv\left[\theta_{k}(x)\right]^{n+1} P_{n, m, k}(x)
$$

We assume that these functions have been normalized so that $c_{n, 0}^{(m, m)}=1$. Denote by $b_{n, s},(n=0,1, \cdots ; s=0,1, \cdots)$, any set of positive numbers, independent of $k$, such that $b_{n, 0}=1$ and

$$
b_{n, s} \geqq\left|\sum_{m=1}^{\nu} c_{n, s-1+\delta(m, k)}^{(m, k)}\right|
$$

for $s>0$, where $\delta(m, k)$ is Kronecker's symbol. Put

* In the work to follow the points $a_{k}$ are assumed finite but the extension to the case where one point $a_{k}$ is the point at infinity is immediate.

$\dagger$ Whittaker and Watson, Modern Analysis, 4th ed., p. 131. 


$$
M_{n}=\max \left(\frac{b_{0, n+1}}{b_{0, n}}, \frac{b_{1, n}}{b_{1, n-1}}, \cdots, \frac{b_{n-1,2}}{b_{n-1,1}}\right) .
$$

Using the above notation we shall now state the principal result of this paper.

THEOREM 1. Let the above number $R$ be such that

$$
0<R<\liminf _{n \rightarrow \infty}\left[b_{n, 1}+M_{n}\right]^{-1},
$$

and such that

$$
\left|P_{n, m, k}(x)\right| \leqq Q
$$

for $x$ on the curves $C_{R^{(k)}}$, where $Q$ is a positive number independent of $n, m$, and $k$. Then if $f(x)$ is analytic at the points $a_{1}, a_{2}, \cdots, a_{\nu}$, it may be expanded in the simple series

$$
\begin{aligned}
f(x) & =\alpha_{0,1} F_{0,1}(x)+\alpha_{0,2} F_{0,2}(x)+\cdots+\alpha_{0, \nu} F_{0, \nu}(x) \\
& +\alpha_{1,1} F_{1,1}(x)+\alpha_{1,2} F_{1,2}(x)+\cdots+\alpha_{1, \nu} F_{1, \nu}(x)+\cdots .
\end{aligned}
$$

This expansion will converge absolutely and uniformly to $f(x)$ in and on the curves $C_{r}{ }^{(k)}$ provided $r \leqq R$ and $r<\rho$, where $\rho$ is any number such that $f(x)$ is analytic in and on every $C_{\rho}{ }^{(k)}$. There is moreover only one such expansion for $f(x)$ which is uniformly convergent in $C_{R^{\prime}}{ }^{(k)}, R^{\prime}>0$.

There are numerous examples in the literature of expansions of functions analytic in distinct regions. ${ }^{*}$ There are fewer instances of expansions like those in Theorem 1, where an arbitrary function analytic at several points can be expanded in the neighborhood of these points in a set of functions independent of that neighborhood. The Jacobi polynomials $\dagger$ furnish the simplest example of such an expansion. Other examples have been given by Walsh $\ddagger$ and, for the case of two points, by the writer. $\S$ Theorem 1 includes these previous expansions of the writer's. The relation with the Jacobi polynomials will be discussed in $\S 3$. In the case of a single point, $\nu=1$, Theorem 1 is a

* J. L. Walsh, Interpolation and Approximation, Colloquium Publications of this Society, vol. 20, p. 128.

$\dagger$ Alfred Kienast, Inaugural-Dissertation, Zurich (1906).

$\ddagger$ Op. cit., $\$ \S 8.7$ and 8.8 .

$\S$ Annals of Mathematics, vol. 35 (1934), pp. 759-766. 
new generalization of Bürmann's series. For $\nu=1, \theta_{1}(x)=x$, it reduces to a theorem recently proved by Gertrude S. Ketchum which, in turn, includes numerous theorems of other writers. ${ }^{*}$

In the above theorem, condition (4) is stated in terms of bounds on the coefficients $c_{n, 8}^{(m, k)}$. In some applications it is more convenient to have all the conditions stated in terms of a bound on the functions $P_{n, m, k}(x)$. We therefore let $M_{n, \sigma}$ be such that

$$
\left|P_{n, m, k}(x)-c_{n, 0}^{(m, k)}\right| \leqq M_{n, \sigma}
$$

for $x$ in and on the curves $C_{\sigma}^{(k)}$. If $l_{\sigma}$ is the maximum length of the analytic curves $C_{\sigma}{ }^{(k)}$, and $T_{\sigma}$ is the upper bound of $\left|\theta_{k}^{\prime}(x)\right|$ for $x$ on $C_{\sigma}{ }^{(k)}$, then it is easily seen that

$$
\left|c_{n, s}^{(m, k)}\right| \leqq K_{\sigma} M_{n, \sigma} \sigma^{-s}, \quad K_{\sigma}=\frac{l_{\sigma} T_{\sigma}}{2 \pi \sigma} .
$$

Substituting this condition in Theorem 1 we get immediately the following corollary.

Corollary $\dagger$ 1. The function $f(x)$ defined above may be expanded in one and only one series (6) which converges absolutely and uniformly in and on the curves $C_{R}{ }^{(k)}$, where $0<R<\rho$ and

$$
R<\sigma\left\{1+K_{\sigma}[\sigma(\nu-1)+1] \limsup _{n \rightarrow \infty} M_{n, \sigma}\right\}^{-1} .
$$

Theorem 1 and Corollary 1 become considerably simpler if we are interested only in the existence of the expansion in the neighborhood of the points $a_{k}$. We now state fully this special case as a second corollary.

Corollary 2. Let $f(x)$ be any function analytic at the distinct points $a_{1}, a_{2}, \cdots, a_{\nu}$. Let $F_{n, m}(x)$ be analytic in a set of regions $R_{k}$, $(k=1,2, \cdots, \nu)$, where $R_{k}$ is independent of $n$ and $m$ and contains $a_{k}$ in its interior. Also let $F_{n, m}(x)$ have a zero of order $n$ at $a_{k}$

* Gertrude S. Ketchum, Transactions of this Society, vol. 40 (1936), p. 213.

$\dagger$ This reduces for $\nu=1$ and $\theta_{1}(x)=x$ to a theorem of Takenaka, Tokyo Physico-Mathematical Society Proceedings, (3), vol. 13 (1931), pp. 111-117. See also I. M. Sheffer, American Journal of Mathematics, vol. 57 (1935), pp. $587-614$. 
if $m=k$ and a zero of higher order than $n$ if $m \neq k$. Suppose a function $\theta_{k}(x)$ exists such that (1) $\theta_{k}(x)$ has a simple zero at $a_{k}$, (2) $\lim _{x \rightarrow a_{k}} F_{n, k}(x)\left[\theta_{k}(x)\right]^{-n}=1$, and (3) $\left|F_{n, m}(x)\left[\theta_{k}(x)\right]^{-n}\right|$ is bounded in $R_{k}$ by a constant $Q$ independent of $n, m$, and $k$. Then there exists a unique set of numbers $\alpha_{n, m}$ such that the series in (6) converges absolutely and uniformly to $f(x)$ in each of a set of regions $R_{k}^{\prime}$, $(k=1,2, \cdots, \nu)$, where $R_{k}^{\prime}$ contains $a_{k}$ in its interior.

2. Proof of Theorem 1. Consider first the expansion of the particular functions $f_{p}(x),(p=0,1, \cdots)$, where

$$
f_{p}(x)=B_{p, k}\left[\theta_{k}(x)\right]^{p}
$$

in some neighborhood of each of the points $a_{k}$. The $B_{p, k}$ are constants. Proceeding formally, writing $F_{n, m}(x)$ in powers of $\theta_{k}(x)$, and equating coefficients, we obtain the following recurrence relation on the coefficients of (6), which we shall call $\alpha_{n, m}^{(p)}$ :

$$
\begin{aligned}
\alpha_{n, m}^{(p)} & =0, \\
\alpha_{p, m}^{(p)} & =B_{p, m}, \\
\alpha_{p+q, m}^{(p)} & =-\sum_{r=0}^{q-1} \sum_{k=1}^{\nu} c_{p+r, q-1-r+\delta(k, m)}^{(k, m)} \alpha_{p+r, k}^{(p)},
\end{aligned}
$$

for $q=1,2, \cdots$.

Let $A_{p+q}^{(p)}$ be a set of numbers defined by the relations

$$
A_{p}^{(p)}=1, \quad A_{p+q}^{(p)}=\sum_{r=0}^{q-1} b_{p+r, q-r} A_{p+r}^{(p)}, \quad(q=1,2, \cdots) .
$$

Let $d_{p}$ be the largest of $\left|B_{m, p}\right|$ for $m=1,2, \cdots, \nu$. Then by induction

$$
\left|\alpha_{p+q, m}^{(p)}\right| \leqq d_{p} A_{p+q}^{(p)}, \quad(q=0,1, \cdots),
$$

for all values of $m$. Now

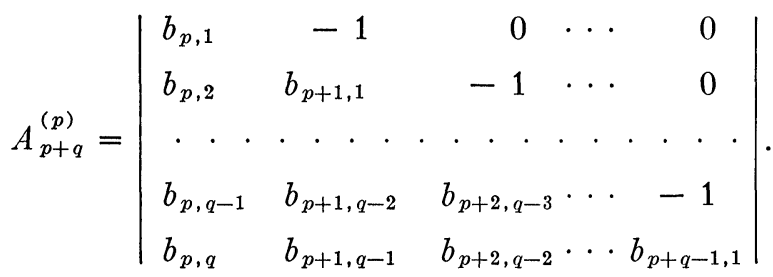


Let

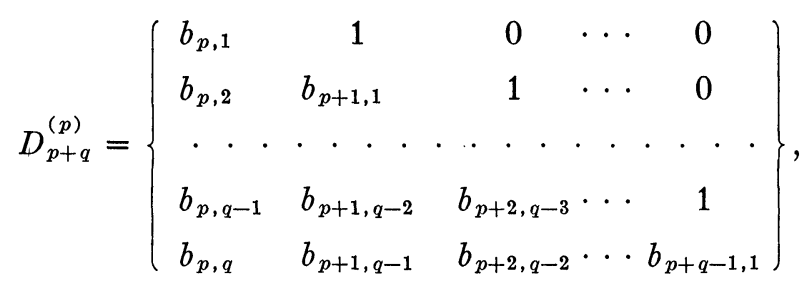

where the bracketed expression is to be expanded like a determinant except that all signs are to be taken positive. Then

$$
D_{p+q}^{(p)} \geqq A_{p+q}^{(p)} \text {. }
$$

Also, by expanding $D_{p+q}^{(p)}$, we find that

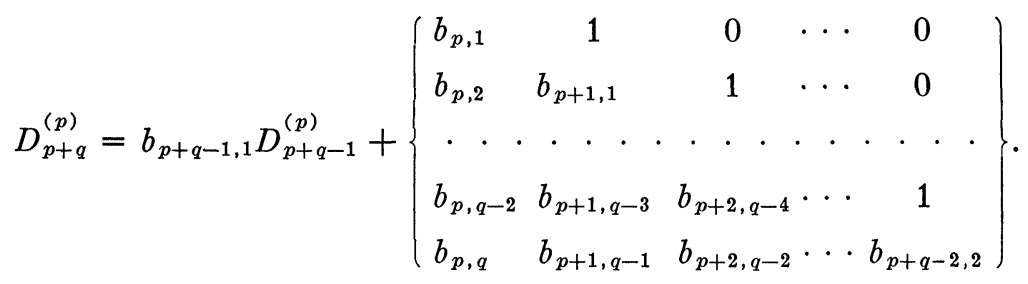

Hence

$$
\begin{aligned}
& D_{p+q}^{(p)} \leqq b_{p+q-1,1} D_{p+q-1}^{(p)}+M_{p+q-1} D_{p+q-1}^{(p)} \\
& D_{p+q}^{(p)} \leqq\left(b_{p+q-1,1}+M_{p+q-1}\right)\left(b_{p+q-2,1}+M_{p+q-2}\right) \\
& \cdots\left(b_{p+1,1}+M_{p+1}\right) b_{p, 1}
\end{aligned}
$$

Now the series

$$
\begin{aligned}
\alpha_{p, 1}^{(p)} F_{p, 1}(x)+\cdots & +\alpha_{p, \nu}^{(p)} F_{p, \nu}(x)+\alpha_{p+1,1}^{(p)} F_{p+1,1}(x)+\cdots \\
& +\alpha_{p+1, \nu}^{(p)} F_{p+1, \nu}(x)+\cdots
\end{aligned}
$$

is dominated by

$$
Q[(\nu-1) R+1] d_{p} R^{p} \sum_{q=0}^{\infty} D_{p+q}^{(p)} R^{q},
$$

for $x$ in and on the curves $C_{R}{ }^{(k)}$. Substituting the bound (11) in (13) we get a series with the ratio of the $(q+1)$ st to $q$ th terms equal to $\left(b_{p+q, 1}+M_{p+q}\right) R$. From (4) it follows that for values of 
$p+q \geqq n_{0}$, this ratio is less than $\lambda<1$. Hence (13) will converge for all $p$ and for $p \geqq n_{0}$ has a sum less than

$$
Q[(\nu-1) R+1](1-\lambda)^{-1} d_{p} R^{p} .
$$

Thus for each $p$, the series (12) converges uniformly for $x$ in and on $C_{R}{ }^{(k)}$. By an extension of the Weierstrass double series theorem we can rearrange the terms in powers of $\theta_{k}(x)$ and verify by means of the recurrence relations (8) that the sum of $(12)$ is $f_{p}(x)$.

Now consider the double series formed by summing (12) for $p=0,1,2, \cdots$. This double series converges absolutely if the series (14) summed over $p$ converges. But this evidently happens if $R<\lim$ inf $d_{p}^{-1 / p}$, which in turn happens if $R<\rho$, which was part of our hypothesis. Hence, since this double series converges absolutely, the terms may be arranged in a simple series so as to yield the expansion (6) of our theorem.

3. Generalization of Expansions in Jacobi Polynomials. In this section we give an illustration of the application of our theory. We obtain a simple class of expansions which are generalizations of expansions in Jacobi polynomials. For simplicity we discuss only the question of the existence of the expansions.

It will be shown that any function $f(x)$ analytic at $a_{1}, \cdots, a_{\nu}$ can be expanded in the series (6), where

$F_{n, m}(x)=\left[\left(x-a_{1}\right)\left(x-a_{2}\right) \cdots\left(x-a_{\nu}\right)\right]^{n+1}\left(x-a_{m}\right)^{-1} G_{n, m}(x)$,

the $G_{n, m}(x)$ being any functions subject to the three conditions: (1) the $G_{n, m}(x)$ are each analytic at $a_{1}, \cdots, a_{\nu},(2) G_{n, m}\left(a_{m}\right) \neq 0$, (3) $\left|G_{n, m}(x)\right|$ is bounded in some set of regions $R_{k}$ about the points $a_{k}$ by a constant independent of $n, m$, and $k$. In particular, these conditions will be satisfied if $G_{n, m}(x) \equiv 1$, in which case the $F_{n, m}(x)$ reduce to the Jacobi polynomials.

Without loss of generality we introduce certain normalizing constants and take $F_{n, m}(x)$ to be

$\frac{\left[\left(x-a_{1}\right)\left(x-a_{2}\right) \cdots\left(x-a_{\nu}\right)\right]^{n+1} G_{n, m}(x)}{\left(a_{m}-a_{1}\right) \cdots\left(a_{m}-a_{m-1}\right)\left(x-a_{m}\right)\left(a_{m}-a_{m+1}\right) \cdots\left(a_{m}-a_{v}\right) G_{n, m}\left(a_{m}\right)}$.

We choose

$$
\theta_{k}(x)=\left(x-a_{1}\right)\left(x-a_{2}\right) \cdots\left(x-a_{\nu}\right) .
$$


Then

$$
\begin{aligned}
& \frac{F_{n, m}(x)}{\left[\theta_{k}(x)\right]^{n}} \\
& =\frac{\left(x-a_{1}\right) \cdots\left(x-a_{m-1}\right)\left(x-a_{m+1}\right) \cdots\left(x-a_{\nu}\right) G_{n, m}(x)}{\left(a_{m}-a_{1}\right) \cdots\left(a_{m}-a_{m-1}\right)\left(a_{m}-a_{m+1}\right) \cdots\left(a_{m}-a_{\nu}\right) G_{n, m}\left(a_{m}\right)} .
\end{aligned}
$$

From this relation it is easily seen that all the conditions of Corollary 2 are satisfied, and our statement follows.

UNIVERSITY OF ILLINOIS

\section{DUALISM IN ABELIAN GROUPS*}

\section{BY REINHOLD BAER}

It has been proved $\dagger$ that a finite Abelian group contains as many subgroups of a given order $n$ as it contains factor groups of order $n$ (=subgroups of index $n$ ) and E. Steinitz $\ddagger$ knew that a finite Abelian group contains as many subgroups of a given structure $\boldsymbol{n}$ as it contains factor groups of structure $\boldsymbol{n}$. It is the aim of this note to prove that such a dualism exists in the Abelian group $G$ if, and only if, $G$ is a group without elements of infinite order whose primary components are finite. This is remarkable as an exception to the rule that every proposition which is satisfied in finite Abelian groups holds also true in every primary Abelian group such that the orders of its elements are bounded.

Let $G$ and $G^{\prime}$ be two (additively written Abelian) groups. Then the function $d$ is a dualism of $G$ upon $G^{\prime}$ if it has the following properties:

(1) $\boldsymbol{d}$ is defined for every subgroup $S$ of $G$ and $S \boldsymbol{d}$ is a uniquely determined subgroup of $G^{\prime}$;

(2) to every subgroup $S^{\prime}$ of $G^{\prime}$ there exists a subgroup $S$ of $G$ such that $S d=S^{\prime}$;

(3) $S \leqq T(\leqq G)$ if, and only if, $T d \leqq S d\left(\leqq G^{\prime}\right)$;

* Presented to the Society, October 31, 1936.

$\uparrow$ Garrett Birkhoff, Subgroups of Abelian groups, Proceedings of the London Mathematical Society, (2), vol. 38 (1934), pp. 385-401.

$\ddagger$ E. Steinitz, Jahresberichte der Deutschen Mathematiker-Vereinigung, vol. 9 (1901), pp. 80-85. 